\title{
Inłaltsüberifiçt
}

\section{Bürgerlictes (Gejebbuct)}

Eriter 9bitinitt. Berionen

\author{
(Exite马 $\mathfrak{B} \mathfrak{u}$ d \\ IrIgemeinter teil . . . . . . . 1
}

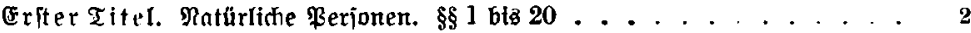

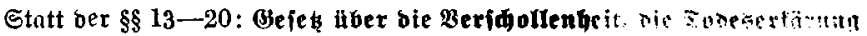
unb bie Fefiftellung ber Tobeģzeit bom 7.4.39 . . . . . . . . . . . 8

8 reiter Titel. Jurijtijhe Perjonen

I. Bereine . . . . . . . . . . . . . . . . . . . . . . 14

1. Allgemeine Borfưriften. $\$ \$ 21$ biłz 54 . . . . . . . . . . . 14

2. Gingetragene Rereine. $\$ 55$ bis 79 . . . . . . . . . . . . 26

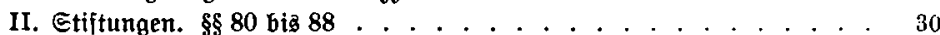

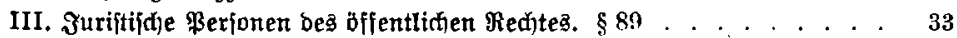

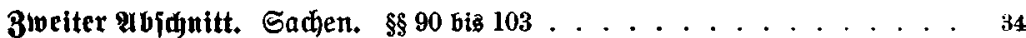

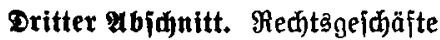

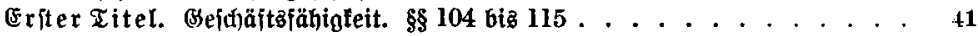

\$reiter ritel. Billenserflärung. $\$ 116$ bỉ $144 \ldots \ldots$. . . . . . . . . 44

Dritter Titel. Bertrag. $\$ 145$ bia 157 . . . . . . . . . . . . . . . 65

Bierter Titel. Bebingung. Bettbeftimmung. $\$ 158$ biz 163. . . . . . . 70

Fünfter Titel. Bertretung. Bollmađt). $\$ 164$ biß $181 \ldots$. . . . . . . 72

Sechiter Titel. Finmilligung. Genehmigung. $\$ 182$ biß 185 . . . . . . $\$ 1$

Bierter 9bidnitt. Frijten. Termine. $\$ \$ 186$ bis $193 \ldots \ldots 3$

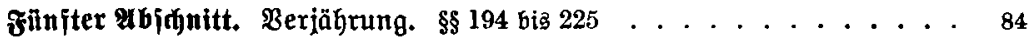

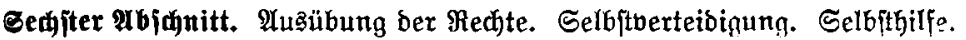
$\$ 226$ bio 231 . . . . . . . . . . . . . . . . . . . . . . . . . . . . 96

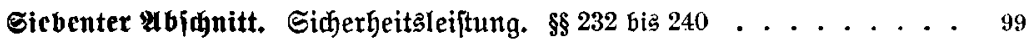

\section{Zroeites $B$ ud \\ Pedut ber Gajuldberhältnifie : . . . . . 101}

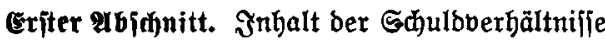

(5rfter Titel. Berpfli(t)tung zur Leiftung. $\$ 241$ biz 292 . . . . . . . . 106

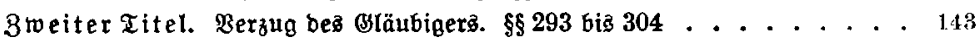

3wciter अbinnitt. Sđulboerbältriffe aus Berträgen

Friter Titel. Begrünoung. 3nhalt bę Bertraģ̧. $\$ 305$ biz 319 . . . . . 146

8 wetter Titel. Begenfeitiger Bertrag. $\$ 3320$ bis 327 . . . . . . . . . 154 


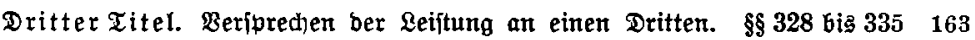

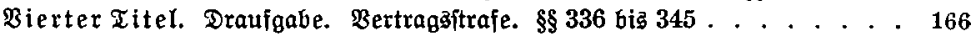

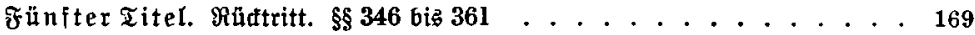

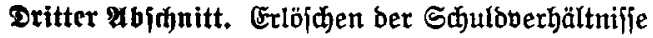

Eriter Titel, Erfüllung. $\$ 362$ biz 371 . . . . . . . . . . . . . 173

3 weiter Titel. Đinterlegung. $\$ \$ 372$ bỉ 386 . . . . . . . . . . . 177

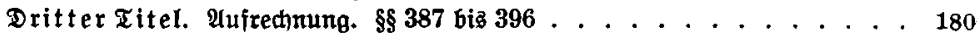

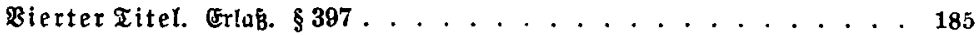

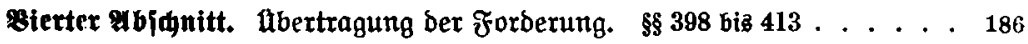

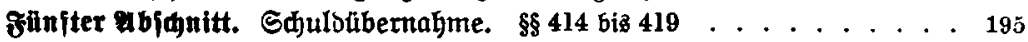

Sedifter Abidnitt. Mehrheit von Sđulonern unb Bläubigern. $\$ 420$ bis 432200

Giebenter abidnitt. Cinzelne Săulbverbältniffe

Erfter Iitel. Sauf. Iaujd) . . . . . . . . . . . . . . . . 206

I. Algemeine Borfthriften. $\$ 433$ biz 458 . . . . . . . . . . . . . 206

II. Bemährletftung wegen Mängel ber Sađje. $\$ 459$ biß $493 \ldots \ldots$. . . . 217

III. Bejonbere Arten bes Raufes

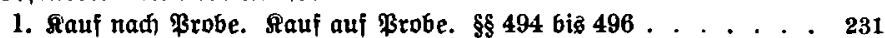

2. \$iebertauf. $\$ 497$ bis 503 . . . . . . . . . . . . . 232

3. Bortauf. $\$ 504$ bis 514 . . . . . . . . . . . . . . . . . 234

IV. Tauff. $\$ 515$. . . . . . . . . . . . . . . . . . . 237

3weiter ittel. Ghientung. $\$ \$ 516$ bis 534 . . . . . . . . . . . . 237

Dritter Titel. Miete. Baht . . . . . . . . . . . . . . . . 244

I. Mitete. $\$ \$ 535$ bio 580 . . . . . . . . . . . . . . . . . . . . . 248

II. ßadt. $\$ 581$ biß 597 . . . . . . . . . . . . . . . . . . . . 271

Bierter Titel. Seihe. $\$ 598$ biz 606 . . . . . . . . . . . . . . 275

Fünfter Titel, Darlehen. $\$ \$ 607$ biß 610 . . . . . . . . . . . . . . 277

Secfter Titel. Dienitwertrag. $\$ 611$ big $630 \ldots \ldots$. . . . . . . . . . 281

Stebenter Titel. Mertvertrag. $\$ 631$ bis 651 . . . . . . . . . . . 301

Qd̆ter Ittel. Mällervertrag. $\$ 652$ biß 656 . . . . . . . . . . . . . . 313

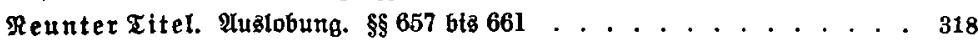

Behnter Xitel. Autrag. $\$ \$ 662$ bia 676 . . . . . . . . . . . . . . . 319

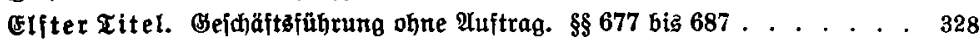

3wölfter Eitel. Berwahmung. $\$ \$ 688$ bis 700 . . . . . . . . . . 332

Dreizehnter Titel. Einbringung von Sadjen bei Baftwirten. $\$ 701$ bis $704 \quad 335$

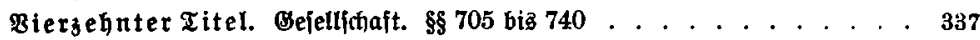

Funfzehnter Iitel. Genteiníd)aft. $\$ \$ 741$ bis 758 . . . . . . . . . . . 351

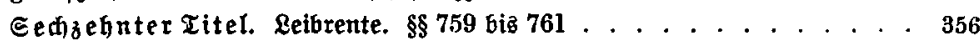

Giebzehnter Ittel. Epiel. Wette. $\$ \$ 762$ bis $764 \ldots \ldots . . . . . . . .357$

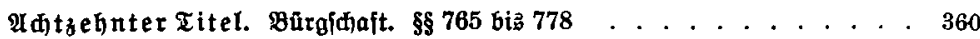

Reunzefnter Titel. Bergleid. $\$ 779$. . . . . . . . . . . . . . . . 369

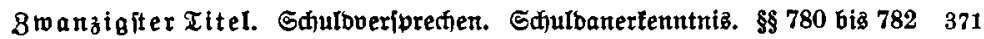

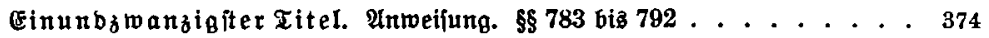

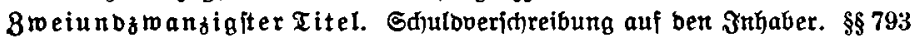

biz 808 . . . . . . . . . . . . . . . . . . . 377

Dreiunbowanzigiter Titel. Borlegung vont Sachen. $\$ 809$ bt3 811 . . 383

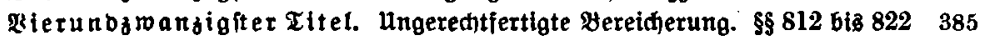

§ânfunbzwanztgfter Titel. Unerlaubte \$anblungen. $\$ \$ 823$ bis 853 . 400 


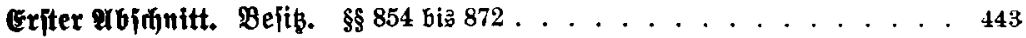

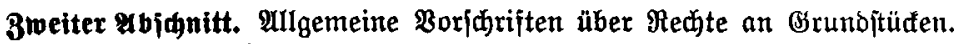

$\$ 873$ bis $902 \ldots \ldots . \ldots \ldots 51$

Oritter qbiditt. Eigentum

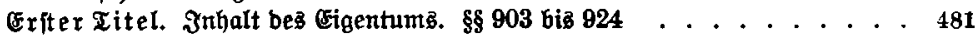

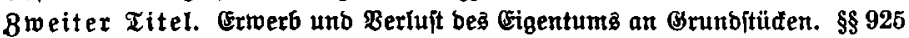

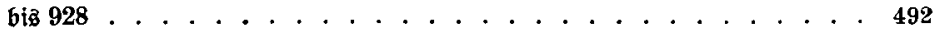

Dritter Titel. Ertwerb unb Berluit bes Cigentums an betweglichen Sachen 498

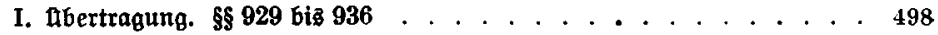

II. Eritgung. $\$ \$ 937$ bis $945 \ldots \ldots \ldots \ldots \ldots$

III. Berbinbung. Bermifatung. Berarbettung. $\$ \$ 946$ bis $952 \ldots \ldots . .506$

IV. Ermerb von Erzeugnifien unb fonjtigen Beftanbteilen eirier Garfe. $\$ \$ 953$ bib $957 \ldots \ldots \ldots \ldots$. . . . . . . . . . . 510

V. $\mathfrak{A n}$ neignung. $\$ 958$ bį $964 \ldots \ldots \ldots \ldots$

VI. $\{u n b . \$ \$ 965$ bis $984 \ldots \ldots \ldots$

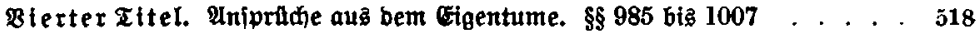

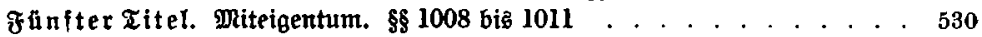

Bierter \$bidnitt. Grbbauredjt. $\$ 1012$ bis 1017 . . . . . . . . . . . . 532

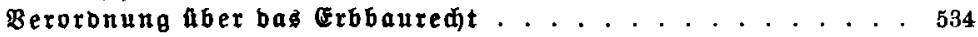

Finfitcr 9bidnitt. Dienjtbarfeiten

Eriter Iitel. Grunbbienţbarteiten. $\$ 1018$ bis $1029 \ldots \ldots$. . . . . . 543

8weiter Titel. Riebbraud . . . . . . . . . . . . 549

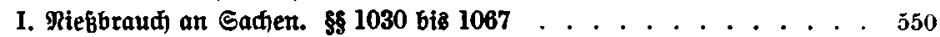

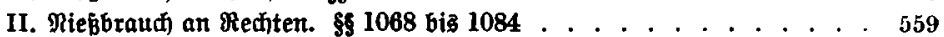

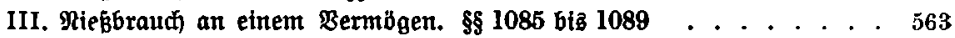

Dritter Titel. Befăräntte perjönliđje Dienftbarteiten. $\$ 1090$ biß̨ 1093 . . 565

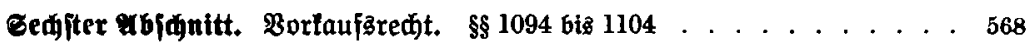

Bicbentex qbidnitt. Reallaften. $\$ 1105$ bis $1112 \ldots \ldots$. . . . . . . 572

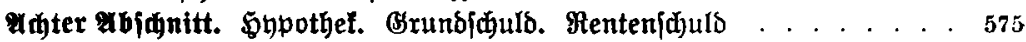

Eriter Ittel. \$ypothef. $\$ 1113$ bis $1180 \ldots \ldots$. . . . . . . . 582

8 weiter Iitel. Brunbidulb. Bentenifulo

I. \$runbifitulb. $\$ 11191 \mathrm{bi} 11198 \ldots \ldots \ldots$

II. Rentenjđulb. $\$ 1199$ biz $1203 \ldots \ldots$. . . . . . . . . 633

Reunter \$bidnitt. Bandredt an betweglidjen Sadjen und an Redten 634

Erfter Iitel. Bfantoredit an berweglichen Sacher. $\$ 1204$ bis $1272 \ldots \ldots 65$

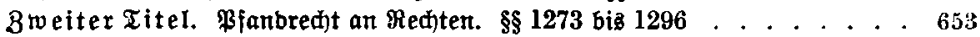

Biertes $\mathfrak{B u}$

Familienredt. . . . . . . 663

Eriter \&binnitt. Bürgerlidhe (Eh)

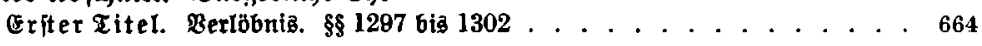

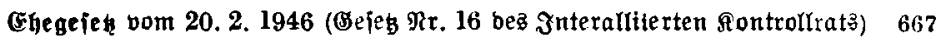

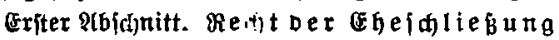

A. Ehefähiqfeit. $\$ 1$ bił $3 \ldots \ldots \ldots 68$

B. Gheverbote. $\$ \$ 4$ bis $10 \ldots \ldots \ldots$ 


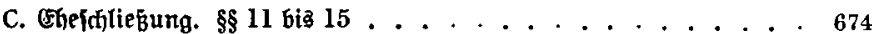

D. Mintigteit ber (che. $\$ \$ 16$ bis 27 . . . . . . . . . . . . . 678

E. Aufhebung ber ohe. $\$ 28$ bis 37 . . . . . . . . . . . . . . 688

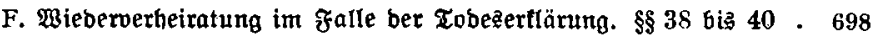

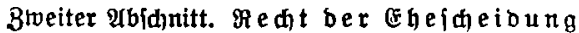

A. Ullgemeine Borjariften. $\$ 41$. . . . . . . . . . . . . . . 703

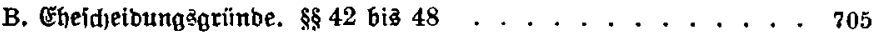
4. Durchührungsెveroronung ३um (E)egefeg. Bom 25. 10.41 (\$\$ 2, 3) 720

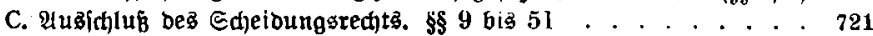

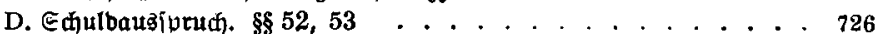

E. Folgen ber €丸)eibung. $\$ 54$ biö 75 . . . . . . . . . . . . 729

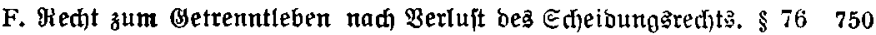

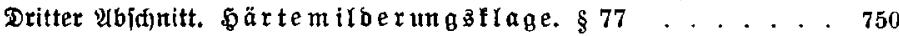

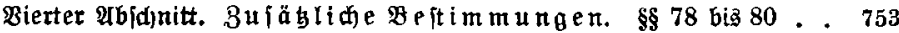

\section{Qrthang zum (Shegeienz:}

1. Beroronung zur Durhführung unb Ergänzung bes Bejebes zur ber.

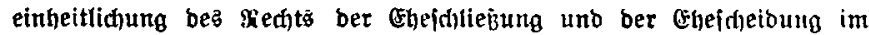

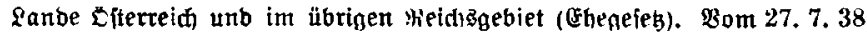

5. Durffithrungaveroronung औum Ehegeleb. Bom 18.3.43........

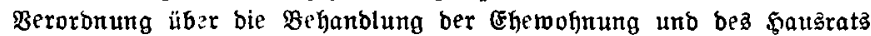
nach ber Sdjeibung (6. DurhfiBD.z. (Ehegefeg). Bom 21.10.44... 761

8 weiter Titel. Fingehung ber the. $\$ 1303$ bis $1322 \ldots \ldots 6$

Dritter Ittel. Ridtigfeit und Anfedtbarfeit ber Che. $\$ 1323$ biz 1347 . $\quad 770$

Bierter Titel. Wieberverheiratung im Falle ber $\mathfrak{z o b e s ̧ e r t l a ̈ r u n g . ~} \$ \$ 1348$

biz 1352 ....................... . 774

Flinfer Titel. Wirfungen ber Che im allgemeinen. $\$ 1353$ bia 1362. . $\quad 775$

Gechiter Titel. Shetidiegి Süterred)t . . . . . . . . . . . . . . 786

I. (b) eqeblithes (süterrect)t

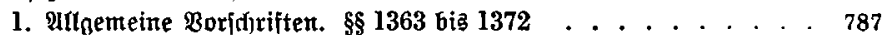

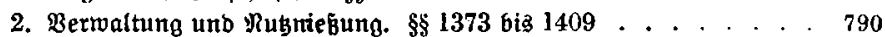

3. Єđ)ulbenhaitung. $\$ \$ 1410$ bis 1417 . . . . . . . . . . . 804

4. Beenbigung ber Berroaltung uno Rusniebung. $\$ \$ 1418$ biş $1425 \quad 806$

5. Gütertrennung. $\$ 1426$ bis 1431 . . . . . . . . . . . . . . 809

II. Bertragsimäß̈ige (Süterrecht

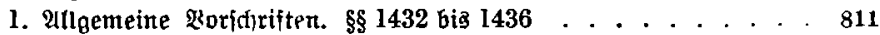

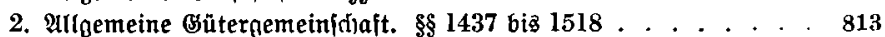

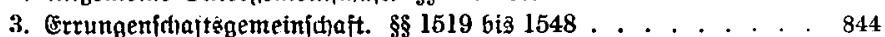

4. Fahniŝgemeinjthaft. $\$ 1549$ biş 1557 . . . . . . . . . . . 851

III. \&üterre(t)täregifter. $\$ \$ 1558$ biฐ $1563 \quad$. . . . . . . . . . . . 853

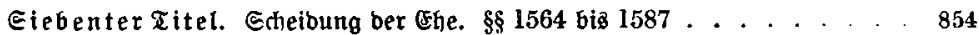

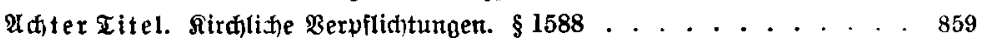

Bociter \&bidnitt. Bertoanotichaft

(5riter Titel. Mrlgemeine Borjutiften. $\$ \$ 1589,1590$. . . . . . . . . 859

8weiter Titel. Ehelithe Ubitammung. $\$ \$ 1591$ bio 1600 . . . . . . . . . 859

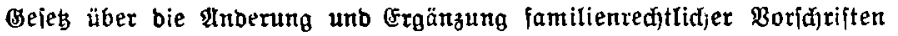
ujto. Bom 12. 4. 38. (\$\$ 26ff.) . . . . . . . . . . . . . . . . . $\$ 60$

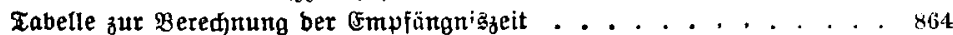


Seite

Dritter Eltel. unterfaltæ̋pflicht. $\$ \$ 1601$ biß̧ $1615 \ldots \ldots 8$

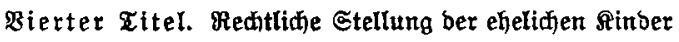

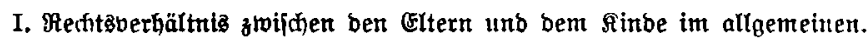

$\$ \$ 1616$ bi 1625 . . . . . . . . . . . . . . . . . . 876

II. Elterliate Getwalt. $\$ 1626$. . . . . . . . . . . . . . 880

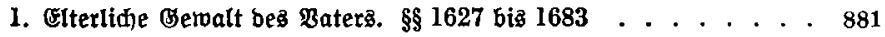

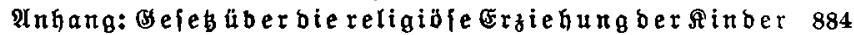

2. Giterliche Gewalt ber Mutter. $\$ 1684$ biz 1698 . . . . . . . 910

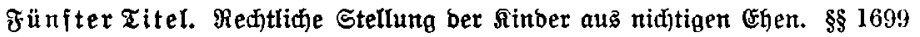
bis 1704 . . . . . . . . . . . . . . . . . . . . . . 916

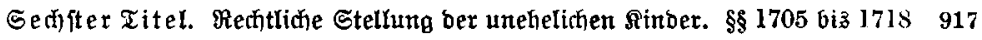

Siebenter Titel. Qegitimation unehelifier Rinber

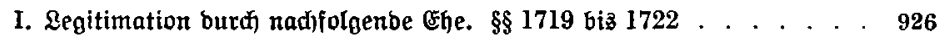

II. Ebelid)teiţerflärung. $\$ \$ 1723$ bis $1740 \ldots \ldots$. . . . . . . . . . . . 928

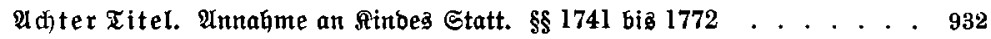

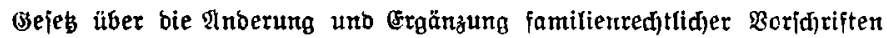

ujw. Bom 12.4.38. (\$\$ 12 fi.) . . . . . . . . . . . . . . . . 943

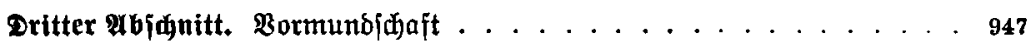

Erifter It el. Bormunbihaft über Minberiährige

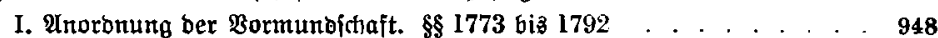

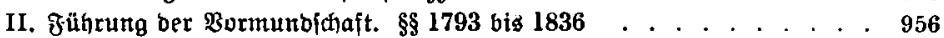

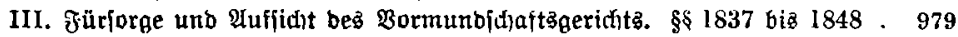

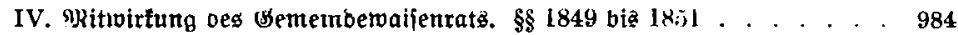

V. Befreite Bormundjchaft. $\$ 1852$ biß 1857 . . . . . . . . . . . . 985

VI. Familienrat. $\$ \$ 1858$ biš 1881 . . . . . . . . . . . . . . . . . . 987

VII. Beentigung ber Bormunbiđlaft. $\$ 1882$ bi 1895 . . . . . . . . . 990

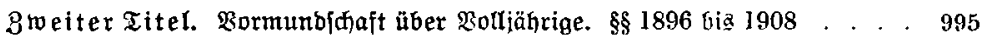

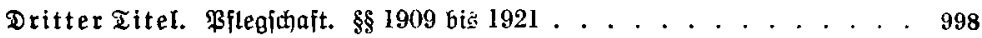

\section{$\mathfrak{F} \mathfrak{u} \mathfrak{n} \mathfrak{f}$ tes $\mathfrak{B} \mathfrak{u} \mathfrak{h}$}

(Erbrent $\ldots \ldots \ldots \ldots \ldots 1006$

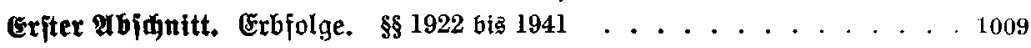

3weiter abidnitt. Rechtliche Stellung bes Erben . . . . . . . . . . . . 1016

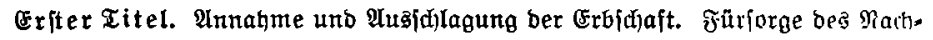

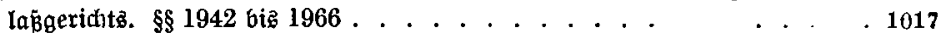

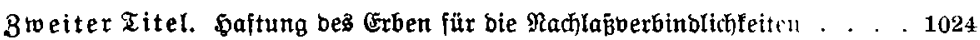

I. Tad)lāberbindlictfeiten. $\$ \$ 1967$ bis 1969 . . . . . . . . . . 1025

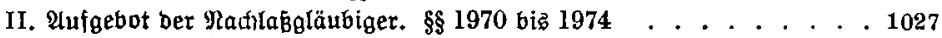

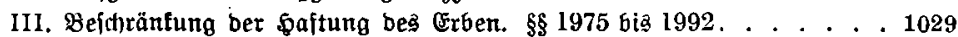

IV. Inventarerriđ)tung. Unbefđ)räntte \$aitung bez (Erben. $\$ \$ 1993$ bis 2013103 (;

V. 2tufjitiebende Cinreben. \$\$ 2014 bis 2017 . . . . . . . . . . . . 1042

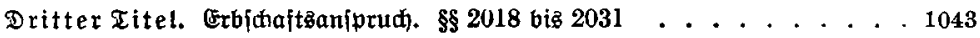

Bierter Iitel. Mehrheit bon Erben . . . . . . . . . . . . . . 1047

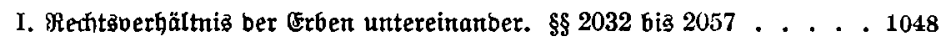

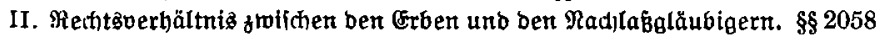

bis 2063 . . . . . . . . . . . . . . . . . 1059 
Selte

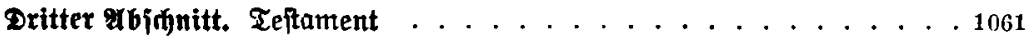

Eriter Titel. Allaemeine Borjđriften. $\$ \$ 2064$ bis̄ $2086 \ldots \ldots$. . . . . . . 1062

\$ 1 weiter Titel. Grbeintébung. $\$ \$ 2087$ bis $2099 \ldots \ldots$

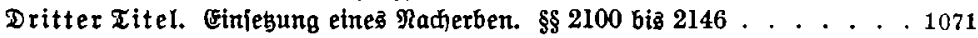

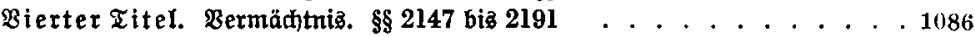

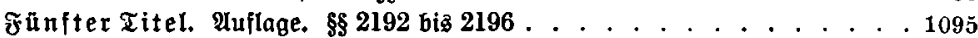

Seniter Iitel. Teitamentşollftređer. $\$ \$ 2197$ biz 2228 . . . . . . . . . 1096

Siebenter Titel. Errichtung unb Nufhebung eines Teitaments. \$\$ 2229

bỉ 2264 . . . . . . . . . . . . . . . . . . . . . . . . . . . . 1109

Geíeg uber bie Crriatung von Teftamenten unb Grbuerträgen. Bom

31.7.38 . . . . . . . . . . . . . . . . . . . 1118

Ad)ter Titel. Bemeiniđaftlidhes Teltament. $\$ \$ 2265$ biz 2273 . . . . . . 1142

Bierter qujunitt. Erbbertrag. $\$ 2274$ bi $2302 \ldots \ldots$. . . . . . . . . . 1146

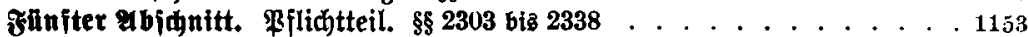

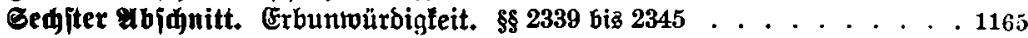

Giebenter \&bidnitt. Erbverzidt. $\$ 2346$ bis 2352 . . . . . . . . . . . . 1167

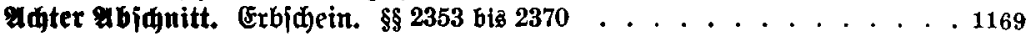

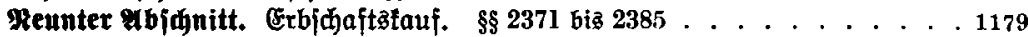

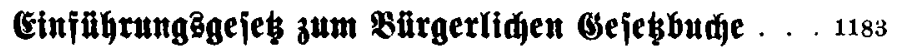

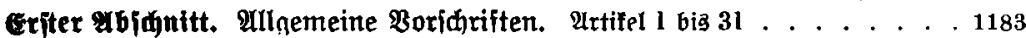

in

Anhang: Deutides $\mathfrak{a n t e r l o f a l e s}$ Brivatredt.

1. Beroronung über bie Deutihe Staatşangefürigfett. Bom 5.2.34 1205

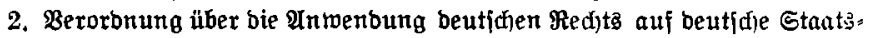
angehörige im ßroteftorat $\mathfrak{B a ̈ h m e n ~ u n b ~ M a ̈ h r e n . ~ B o m ~ 2 0 . 7 . 3 9 ~ . ~} 1206$

3. Berorbnung über bie beutidie Serid)t\$barfeit im Beneralgoubernement. Bom 19.2.40 . . . . . . . . . . . . . . 1207

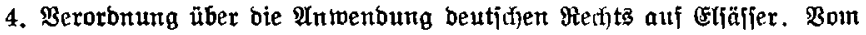

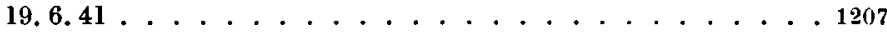

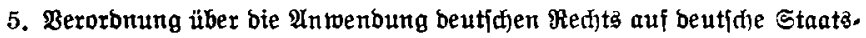
angehörige in ben bcjekten Ditgebieten. Bom 27.4.42 . . . . 1208

6. Berorbnung über ben $\mathfrak{A n}$ wenbungäberei(f) erbre(f)tlifjer Boridrifter. Bom 12.12.41 . . . . . . . . . . . . . . . . 1209

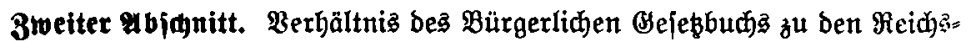

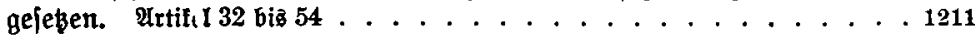

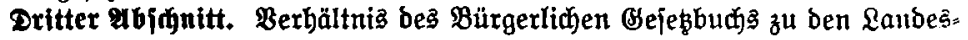

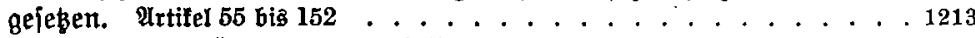

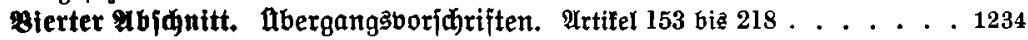
$\mathfrak{A} \mathfrak{n} \mathfrak{h} \mathfrak{a} \mathfrak{g}$.

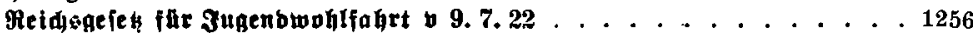

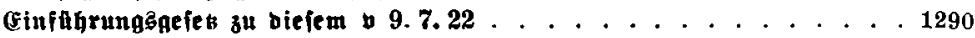

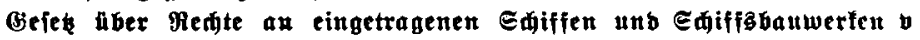

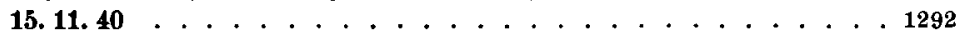

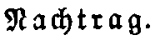

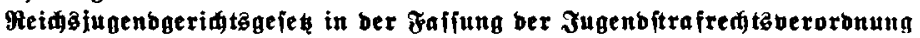

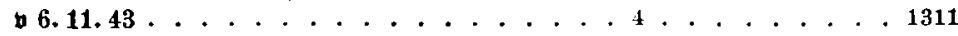

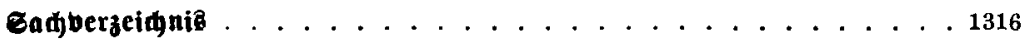

